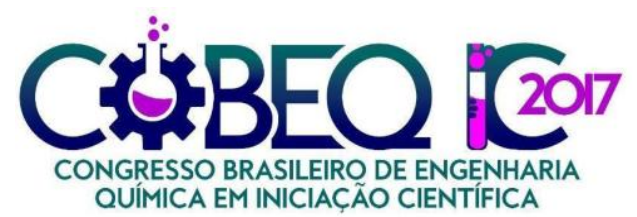

\title{
DESENVOLVIMENTO DE UM MODELO MATEMÁTICO PARA A DETERMINAÇÃO DO CALOR ESPECÍFICO DE SOLUÇÕES DE LEITE/SACAROSE EM FUNÇÃO DA TEMPERATURA E DA COMPOSIÇÃO.
}

\author{
T. S. TOMASELLO ${ }^{1}$, W. G. RODRIGUES ${ }^{1}$, G. R de CARVALHO ${ }^{1}$ e J. TELIS-ROMERO ${ }^{1}$ \\ ${ }^{1}$ Universidade Estadual Paulista, Departamento de Engenharia e Tecnologia de Alimentos \\ E-mail para contato: thais.siqueira.tomasello@gmail.com
}

\begin{abstract}
RESUMO - O presente trabalho de pesquisa apresenta os termogramas de misturas de leite semidesnatado e sacarose como função da fração de sólidos e da temperatura. $\mathrm{O}$ calor específico foi determinado experimentalmente usando um calorímetro diferencial analítico (DSC). Os resultados experimentais foram simulados usando modelos empíricos de primeira ordem, através do uso de um algoritmo não linear com subsequente análise estatística. Todos os modelos mostraram um bom comportamento frente aos resultados experimentais, mostrando valores do coeficiente de correlação ajustado superior a 0,98. Os resultados obtidos nesta pesquisa podem proporcionar informações úteis para a modelagem, desenho e controle das operações unitárias envolvidas na indústria de leite condensado.
\end{abstract}

\section{INTRODUÇÃO}

A produção mundial de leite em 2013 foi de 552.091.000 (quinhentos e cinquenta e dois milhões e noventa e um mil) toneladas, o que representou um crescimento global de $7,33 \%$ da produção de 2010. (USDA, 2013).

O Brasil é o maior produtor de leite da América Latina, com 32.380 mil toneladas, Com essa produção, o Brasil ocupa o quinto lugar no ranking mundial de produtores de leite, perdendo apenas para União Europeia, Índia, Estados Unidos e China (USDA, 2013). Parte da produção mundial de leite é utilizada para a preparação do leite condensado, leite em pó e leite evaporado.

Com relação ao leite condensado, em 2016, o Brasil exportou cerca de $27 \%$ do valor total exportado de derivados lácteos. Já o leite em pó, é o líder na exportação representando $55,6 \%$ do valor total (CONAB, 2016).

Para determinação do calor específico é necessário conhecer as propriedades termo físicas do leite/sacarose, pois é muito importante para o projeto, simulação, otimização e operações unitárias como a evaporação e trocadores de calor. O calor específico $(\mathrm{Cp})$ de uma substância é uma propriedade termodinâmica fundamental que tem importância em todos os 
processos envolvendo a captação, libertação e transferência de energia térmica. Especificamente na operação unitária de evaporação, o $\mathrm{Cp}$ é importante no projeto e evaporadores de múltiplo efeito uma vez que o seu valor altera, principalmente, com a concentração de soluto.

Embora algumas propriedades do leite se encontrem listadas na literatura, como calor específico, viscosidade dinâmica, condutividade térmica e massa específica (MINIM et al, 2002), dados de calor específico de misturas de leite e sacarose ainda não estão disponíveis na literatura especializada. Portanto, o conhecimento dessa propriedade desperta um grande interesse na indústria de produtos concentrados de leite. Neste trabalho foi determinado o calor específico do leite, em função da sua concentração de sólidos totais.

\section{METODOLOGIA}

\subsection{Material}

Para a preparação das amostras dos experimentos, foram adquiridos, num supermercado local de são José do rio preto, 24 litros de leite semi-desnatado pasteurizados e homogeneizados e 20 quilogramas de açúcar cristal ambos materiais de um mesmo lote.

A composição do leite foi determinada pelas seguintes análises oficiais, seguindo a metodologia proposta pelo Instituto Adolfo Lutz (2008): gorduras, método de Gerber, proteínas método de Kjeldahl clássico, água método de determinação do extrato seco total e cinzas método de incineração. Carboidratos foram determinados por diferença. A composição do leite semidesnatado com 8 repetições encontra-se na Tabela 1, encontram-se os valores do desvio das repetições.

Tabela 1. Composição de leite semidesnatado pasteurizado e homogeneizado.

\begin{tabular}{lrrrrrrrrrr}
\hline & & \multicolumn{1}{c}{ Amostra } & & & \\
\hline $\begin{array}{l}\text { Compone } \\
\text { ntes }\end{array}$ & 1 & 2 & 3 & 4 & 5 & 6 & 7 & 8 & média & Sy \\
\hline Água & & & & & & & & & & \\
& 89,575 & 89,58 & 88,33 & 88,91 & 88,91 & 88,72 & 88,38 & 88,98 & 89,052 & 0,437 \\
Proteína & 3,849 & 3,849 & 3,687 & 3,708 & 3,704 & 3,714 & 3,741 & 3,728 & 3,747 & 0,065 \\
Carboidrato & 3,946 & 3,944 & 5,395 & 4,770 & 4,767 & 4,959 & 4,276 & 4,680 & 4,592 & 0,505 \\
$\quad$ Gordura & 1,686 & 3,944 & 1,649 & 1,668 & 1,669 & 1,663 & 1,658 & 1,667 & 1,668 & 0,013 \\
$\quad$ Cinzas & 0,944 & 0,938 & 0,936 & 0,941 & 0,941 & 0,939 & 0,937 & 0,943 & 0,940 & 0,003 \\
\hline
\end{tabular}

\subsection{Preparação das amostras para o equipamento DSC}

Para a determinação do calor específico das misturas leite/sacarose foram preparadas 11 amostras com diferentes concentrações, a partir de amostras iniciais com $92 \%$ em peso de leite semidesnatado e $8 \%$ de sacarose. O procedimento para a obtenção dessas concentrações foi realizado utilizando um Evaporador Rotativo a Vácuo, marca MARCONI, modelo MA 120/TH, com capacidade até $2500 \mathrm{ml}$. As misturas leite/sacarose foram submetidas a diferentes etapas de evaporação, obtendo-se diferentes composições determinadas a partir de balanços de massa, como podem ser encontradas na Tabela 2. 
Tabela 2. Onze amostras de diferentes concentrações obtidas por meio de evaporação para obter os calores específicos de leite semidesnatado.

\begin{tabular}{cccccc}
\hline Amostra & Água & Proteína & Carboidratos & Gordura & Cinzas \\
\hline 1 & 81,928 & 3,447 & 12,225 & 1,535 & 0,865 \\
2 & 77,568 & 4,297 & 15,174 & 1,905 & 1,074 \\
3 & 74,279 & 4,906 & 17,399 & 2,184 & 1,231 \\
4 & 70,512 & 5,625 & 19,947 & 2,504 & 1,411 \\
5 & 66,163 & 6,455 & 22,889 & 2,873 & 1,619 \\
6 & 61,588 & 7,328 & 25,984 & 3,261 & 1,838 \\
7 & 56,726 & 8,256 & 29,273 & 3,674 & 2,071 \\
8 & 50,856 & 9,376 & 33,244 & 4,172 & 2,352 \\
9 & 45,003 & 10,493 & 37,203 & 4,669 & 2,632 \\
10 & 37,591 & 11,907 & 42,217 & 5,298 & 2,987 \\
11 & 29,622 & 13,427 & 47,608 & 5,974 & 3,368 \\
\hline
\end{tabular}

Cada mistura leite/sacarose preparada foi colocada em uma panela de alumínio (ref 0219-0062, Perkin Elmer, USA). A panela de alumínio para amostras líquidas foi colocada na balança onde foi adicionado cuidadosamente o volume de $5 \mu \mathrm{L}$ por meio de uma microsseringa de $25 \mu \mathrm{L}$ (Agilent, Santa Clara, USA) e, finalmente, foi tampada hermeticamente.

\subsection{Determinação do calor específico da mistura leite semidesnatado sacarose}

$\mathrm{O}$ calor específico $\left(c p, \mathrm{~kJ}_{\mathrm{kg}}{ }^{-1} .{ }^{\circ} \mathrm{C}\right)$ foi medido por calorimetria diferencial de varredura usando um DSC 8000 (Perkin Elmer, Shelton, USA), nas composições mostradas na tabela 2 e temperaturas de $10,20,30,40,50,60$ e $70{ }^{\circ} \mathrm{C}$. O equipamento DSC foi previamente calibrado com Índio (T.f $156,6^{\circ} \mathrm{C}, \Delta H_{f}=28,45 \mathrm{~kJ} / \mathrm{kg}$ ) a uma taxa de aquecimento de 10 ${ }^{\circ} \mathrm{C} / \mathrm{min}$. Gás nitrogênio $(99,5 \%$ de pureza) foi usado como gás de purga, a um fluxo de $\sim 20 \mathrm{~mL} / \mathrm{min}$. Panelas de alumínio para líquidos voláteis 24,01 $\pm 0.04 \mathrm{mg}$ (ref 0219-0062, Perkin Elmer, USA) foram usadas como linha base e como porta amostra do material de referência (ARCHED, 1993), discos de safira sintética de $3 \mathrm{~mm}$ de diâmetro (ref 0219-1268, Perkin Elmer, Shelton, USA), assim como as amostras leite/sacarose. As amostras foram seladas e pesadas antes de cada procedimento experimental. A linha base, material de referência e amostras foram submetidas ao seguinte programa de temperatura: isotérmico a 0 ${ }^{\circ} \mathrm{C}$ por $4 \mathrm{~min}$, taxa de aquecimento a $10{ }^{\circ} \mathrm{C} / \mathrm{min}$ até $56{ }^{\circ} \mathrm{C}$ e isotérmico a $56{ }^{\circ} \mathrm{C}$ por $4 \mathrm{~min}$, de acordo com a metodologia empregada pela ASTM International(ASTM E1269, 2005). O programa do software PYRIS 11.0 (Perkin Elmer, Shelton, USA) foi usado para analisar e plotar os dados do programa térmico. O calor específico $\left(c_{p}, \mathrm{~kJ} / \mathrm{kg}{ }^{\circ} \mathrm{C}\right)$ das misturas leite/sacarose foi calculado de acordo com a Equação 1:

$$
C p==\frac{D_{s}}{W_{s} \theta}
$$

onde $D_{s}$ representa o deslocamento vertical das curvas térmicas entre a panela da amostra e do 


\section{Congresso Brasileiro de Engenharia Química em Iniciação Científica \\ UFSCar - São Carlos - SP

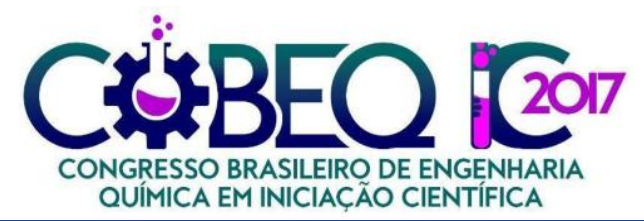

material de referência em um nível de temperatura $(\mathrm{mW}), W_{s}$ é a massa da amostra $(\mathrm{mg})$ e, $\theta$ é a taxa de aquecimento $\left({ }^{\circ} \mathrm{C} / \mathrm{s}\right)$.

\subsection{Análise estatística}

Modelos empíricos foram estabelecidos usando o procedimento "Regression Model Selection" do programa Statgraphics ${ }^{\circledR}$ Centurion XV (Statpoint Technologies Inc., Warrenton, VA, USA), usando equações de primeiro e segundo grau em função da concentração e temperatura. O ajuste do coeficiente de determinação $\left(R_{a d j}^{2}\right)$ e o erro médio relativo (MRE) foram usados para verificar a eficiência dos modelos (Castell-Palou et al. 2012).

$$
\begin{aligned}
& R_{a d j}^{2}=1-\frac{S_{y x}^{2}}{S_{y}^{2}} \\
& M R E=\frac{100}{n} \sum_{i=1}^{n} \frac{\left|c_{p_{i}}-c_{p_{i}}\right|}{c_{p_{i}}}
\end{aligned}
$$

Nas equações 2 e $3, S_{y}$ é o desvio padrão da amostra $(\mathrm{kJ} / \mathrm{kg} \cdot \mathrm{K}), S_{y x}$ é o desvio padrão do modelo $(\mathrm{kJ} / \mathrm{kg} \cdot \mathrm{K}), \hat{c}_{p}$ é o calor específico estimado pelo modelo $(\mathrm{kJ} / \mathrm{kg} \cdot \mathrm{K})$ e $n$ é o número de pontos experimentais usados na análise de regressão.

\section{RESULTADOS}

Foi determinado o calor específico de misturas leite/sacarose na faixa de temperatura de 10 a $70{ }^{\circ} \mathrm{C}$ e concentração de sólidos totais entre 0,18072 e 0,70378 usando o método do DSC. Os resultados experimentais são mostrados na Figura 1.

Figura 1 - Dados experimentais do calor específico (cp) em função da temperatura $T\left({ }^{\circ} \mathrm{C}\right)$ e concentração de sólidos totais $\left(\mathrm{x}_{\mathrm{sT}}\right)$.
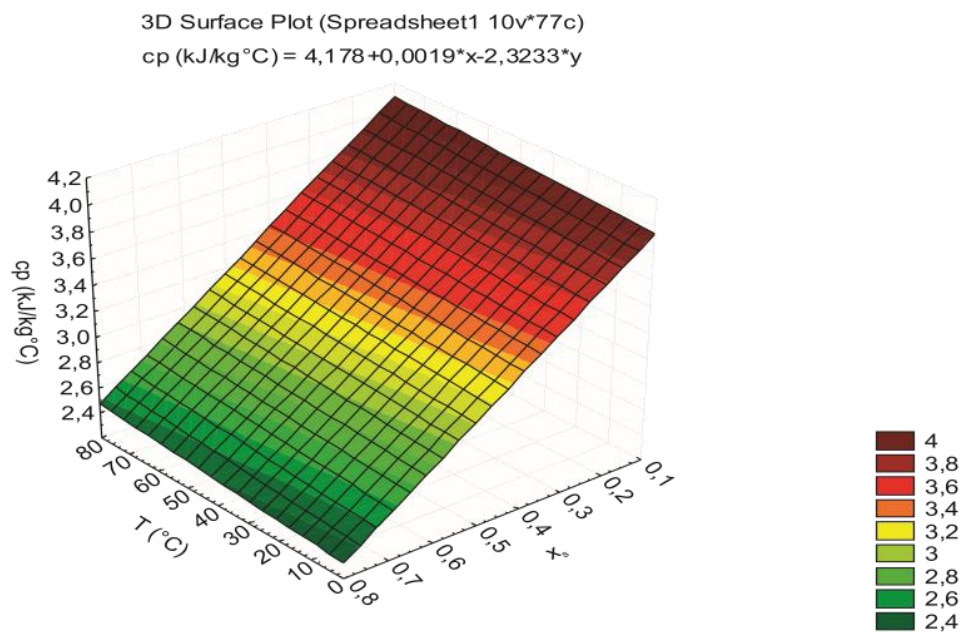
XII Congresso Brasileiro de Engenharia Química

em Iniciação Científica

UFSCar - São Carlos - SP

16 a 19 de Julho de 2017

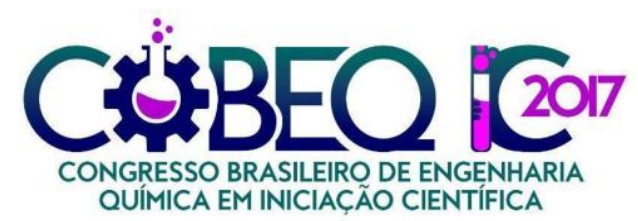
seguir.

A avaliação do modelo empírico de primeiro grau utilizado é apresentada na tabela 2 a

Tabela 2 - Modelo empírico de primeiro grau ajustado ao calor específico cp de mistura de leite/sacarose

\begin{tabular}{lc}
\hline Modelo: $\mathrm{Cp} / \mathrm{kJ} . \mathrm{kg}^{-1} \mathrm{~K}^{-1}=\alpha_{1} \mathrm{~T}_{\mathrm{abs}}+\alpha_{2} \mathrm{X}_{\mathrm{w}}+\alpha_{3}$ \\
\hline $\mathrm{R}^{2}{ }_{\text {adj }}=0,99$ & $\alpha_{1}=0,0019{\mathrm{~kJ} . \mathrm{kg}^{-1} \mathrm{~K}^{-1}}$ \\
$\alpha_{2}=-2,3233 \mathrm{~kJ} . \mathrm{kg}^{-1} \mathrm{~K}^{-1}$ \\
$\alpha_{3}=3,6502 \mathrm{~kJ} . \mathrm{kg}^{-1} \mathrm{~K}^{-1}$ \\
\hline
\end{tabular}

A correlação determinada pode ser utilizada para produção de evaporados e trocadores de calor, pois foi satisfatória utilizando o modelo empírico proposto. A eficiência do modelo pode ser comprovada pelos valores do erro médio relativo $(M R E)$ e do ajuste do coeficiente de determinação $\left(R_{a d j}^{2}\right)$, sendo que o primeiro resultou em $1 \%$ e o segundo em 0,99 .

\section{CONCLUSÕES}

O modelo empírico de primeiro grau foi empregado para simular os dados experimentais do calor específico da mistura de leite/sacarose na faixa de temperatura de 10 a $70{ }^{\circ} \mathrm{C}$, utilizando uma concentração de sólidos totais entre 0,18072 e 0,70378 . Partindo do método do DSC, os resultados obtidos foram satisfatórios visto que na literatura se encontram poucos materiais aplicando a determinação do calor específico mistura de leite/sacarose. Posteriormente há interesse em realizar a correlação do calor específico em função de carboidrato, proteína, gordura e cinzas.

\section{NOMENCLATURA}

Cp: Calor específico $\left(\mathrm{kJ} \cdot \mathrm{kg}^{-1} \cdot \mathrm{K}^{-1}\right)$;

$\hat{c}_{p}$ : calor específico estimado pelo modelo $\left(\mathrm{kJ}^{\mathrm{kg}} \mathrm{kg}^{-1} \cdot \mathrm{K}^{-1}\right)$;

n: número de pontos experimentais usados na análise de regressão;

Sy : Desvio Padrão da amostra $(\mathrm{kJ} / \mathrm{kg} \cdot \mathrm{K})$,

Syx : Desvio padrão do modelo $(\mathrm{kJ} / \mathrm{kg} \cdot \mathrm{K})$;

$\mathrm{T}$ : Temperatura (K);

Xst: Concentração de sólidos totais (\%);

Xw: Concentração de água (\%) 


\section{REFERÊNCIAS}

ARCHED, D. G. 1993. Thermodynamic properties of synthetic sapphire ( $\alpha$-A1203) standard reference material 720 and the effect of temperature-scale differences on thermodynamic properties. J Phys Chem Ref Data. v. 22, p. 1441-1453.

ASTM E1269. Standart test method for determining specific heat capacity by differentail scanning calorimetry. West Conshohocken: ASTM International. 2005.

CASTEll-PAlOU A., Váquiro H.A., Cárcel J.A., Rosselló C., Femenia A., Simal S. (2012). Mathematical modeling of moisture distribution and kinetics in cheese drying. Drying Technology 30 (11-12), 1247-1255.

CONAB - Companhia Nacional de Abastecimento. Disponível em: \&lt;http://www.conab.gov.br/\&gt;. Acesso em: 20 de fevereiro de 2017.

IAL. INSTITUTO ADOLFO LUTZ. Normas Analíticas do Instituto Adolfo Lutz. Métodos químicos e físicos para análise de alimentos. $4^{\mathrm{a}}$ ed. São Paulo: Instituto Adolfo Lutz, 2008 .

MINIM, A. Luis; COIMBRA, S. R. Jane; MINIM, P. R. Valéria; ROMERO, T. Javier. Influence of Temperature and Water and Fat Contents on the Thermophysical Properties of Milk. Journal Of Chemical And Engineering. p. 1488-1491. 25 out. 2002.

USDA - United States Departoneladaent of Agriculture. Disponível em: <https://www.usda.gov/>. Acesso em: 20 de fevereiro de 2017. 